\title{
Domain decomposition based contact solver
}

\author{
J. Dobiáś ${ }^{1}$, S. Pták ${ }^{1}$, Z. Dostál ${ }^{2}$ \& V. Vondrák ${ }^{2}$ \\ ${ }^{1}$ Institute of Thermomechanics, Prague, Czech Republic \\ ${ }^{2}$ University of Ostrava, Ostrava, Czech Republic
}

\begin{abstract}
The paper is concerned with the application of a new variant of the FETI domain decomposition method called the Total FETI to the solution of contact problems by the finite element method. The basic idea is that both the compatibility between adjacent sub-domains and Dirichlet boundary conditions are enforced by the Lagrange multipliers with physical meaning of forces, while the displacements are eliminated. We introduce the Total FETI technique to solve the equations and inequalities governing the equilibrium of system of bodies in contact. Moreover, we show implementation of the method into a code which treats the material and geometric non-linear effects. Numerical experiments were carried out with our inhouse general purpose package PMD.

Keywords: contact, domain decomposition, non-linear, Lagrange multipliers, finite element method.
\end{abstract}

\section{Introduction}

Modelling contact phenomena is still a challenging problem of non-linear computational mechanics. The complexity of such problems arises from the fact that we do not know the regions in contact until we have run the problem. Their evaluations have to be part of the solution. In addition, the solution across the contact interface is non-smooth. In other words, a general contact problem is strongly non-linear and its reasonable solution in terms of a numerical technique, usually the finite element method, needs high quality software stemming from techniques exhibiting qualities like fast convergence rate, good parallel and numerical scalabilities, and so on.

In 1991 Farhat and Roux [1] came up with a novel domain decomposition method called FETI (Finite Element Tearing and Interconnecting method). This 
method belongs to the class of non-overlapping spatial decompositions. Its key concept is based on the idea that satisfaction of the compatibility between spatial sub-domains, into which a domain is partitioned, is enforced by the Lagrange multipliers with physical meaning of forces in this context. After eliminating the primal variables, which are displacements in the displacement based analysis, the original problem is reduced to a small, relatively well conditioned, typically equality constrained quadratic programming problem that is solved iteratively. The CPU time that is necessary for both the elimination and iterations can be reduced nearly proportionally to the number of processors, so that the algorithm exhibits the parallel scalability. This method has proved to be one of the most successful algorithms for parallel solution to problems governed by elliptic partial differential equations. Observing that the equality constraints may be used to define so called 'natural coarse grid', Farhat et al. [2] modified the original FETI algorithm in such a way that they were able to prove its numerical scalability.

The fact that sub-domains act on each other in terms of forces suggests that the FETI approach can also be naturally applied to solution to the contact problems. To this effect the FETI methodology is used to prescribe conditions of non-penetration between bodies. We shall obtain a new minimisation problem with additional non-negativity constraints which replace more complex general nonpenetration conditions; see Dostál et al. [3]. It turned out that the scalability of the FETI methods may be preserved even for solution to the contact problems [3, 4].

A new variant of the FETI method, called the Total FETI (TFETI) method, was presented by Dostál et al. [5]. In this paper we are concerned with application of this method to solution to the contact problems while we in addition consider the material and geometric non-linear effects.

We briefly introduce theoretical foundations of the FETI and TFETI methods. Then we describe an algorithm, in which the TFETI based contact solver accounts for the inner loop, while the outer loop is concerned with the non-linear effects others than the contact. The numerical experiments were carried out with our in-house general purpose finite element package PMD (Package for Machine Design) [6].

\section{Original FETI method}

Consider the static case of a contact problem between two solid deformable bodies denoted as $\Omega_{1}$ and $\Omega_{2}$. We assume that their boundaries are subdivided into three disjoint parts $\Gamma^{u}, \Gamma^{f}$, and $\Gamma^{c}$ with the Dirichlet, Neumann and contact conditions, respectively; see fig. 1(a). The governing equations are given by the equilibrium conditions along with the boundary conditions; see, e.g., Laursen [7] for comprehensive survey of formulations.

Fig. 1(b) shows a discretised version of the contact problem from fig. 1(a). Both bodies, i.e. sub-domains, are discretised in terms of the finite elements method. This figure also shows applied Dirichlet boundary conditions, some displacements, denoted as $u$, and the contact interface. The displacements are the primal variables in the context of the displacement based finite element analysis. 


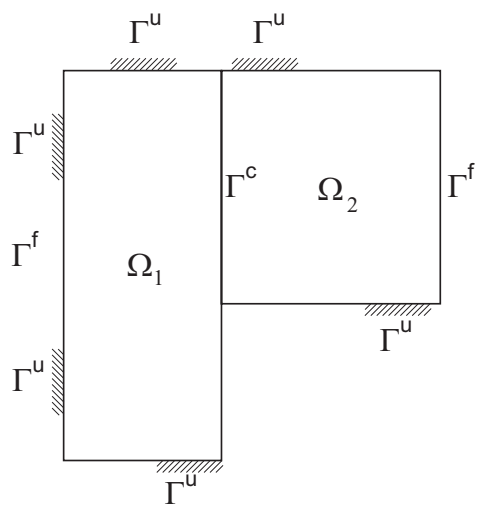

(a) Original problem.

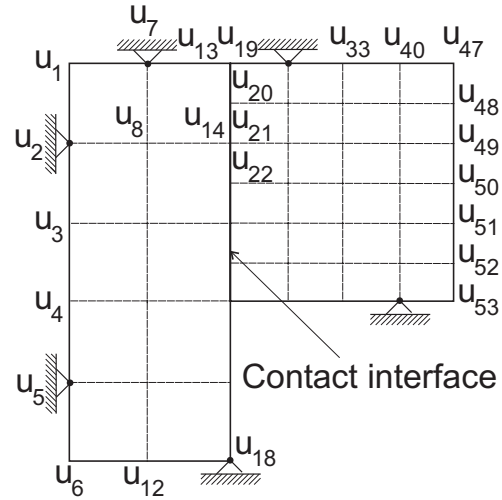

(b) Discretised problem.

Figure 1: Basic notation.

The result of application of the FETI method to the computational model from fig. 1 is depicted in fig. 2(a). The left sub-domain is decomposed into two subdomains with fictitious interface between them. The fundamental idea of the FETI method is that the compatibility between sub-domains along both fictitious and contact interfaces is ensured by means of the Lagrange multipliers with the physical meaning of forces. They are also called the dual variables. $\lambda^{E}$ denotes the forces along the fictitious interface and $\lambda^{I}$ stands for the forces generated by contact.

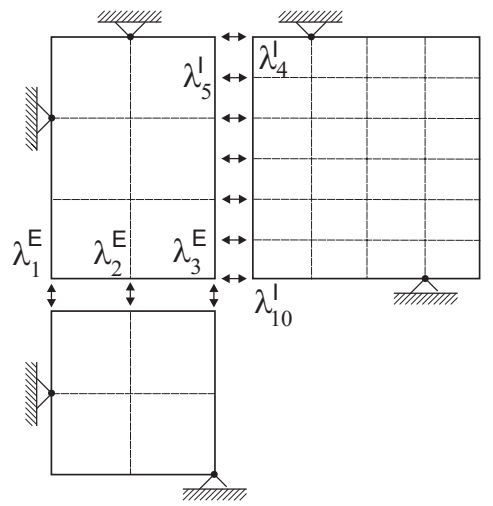

(a) FETI.

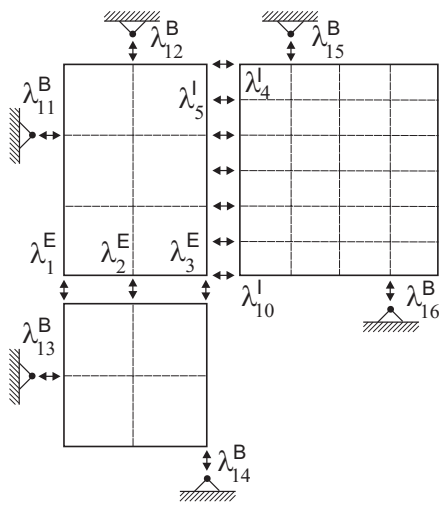

(b) Total FETI.

Figure 2: Principles of FETI and TFETI.

Let $N$ be a number of sub-domains and let us denote for $i=1, \ldots, N$ by $K_{i}, f_{i}$, $u_{i}$ and $B_{i}$ the stiffness matrix, the vector of externally applied forces, the vector of 
displacements and the signed matrix with entries $-1,0,1$ defining the sub-domain interconnectivity for the $i$-th sub-domain, respectively. The matrix $B$ is composed of matrices $B^{I}$ and $B^{E}, B=\left[B^{I} B^{E}\right] . B^{E}$ introduces connectivity conditions along the fictitious interfaces and $B^{I}$ along the contact ones.

The discretised version of the problem is governed by the equation

$$
\min \frac{1}{2} \mathrm{u}^{\top} K \mathrm{u}-f^{\top} \mathrm{u} \quad \text { subject to } \quad B^{I} \mathrm{u} \leq 0 \quad \text { and } \quad B^{E} \mathrm{u}=0
$$

where

$$
K=\left[\begin{array}{ccc}
K_{1} & & \\
& \ddots & \\
& & K_{N}
\end{array}\right], \quad f=\left[\begin{array}{c}
f_{1} \\
\vdots \\
f_{N}
\end{array}\right], \quad u=\left[\begin{array}{c}
u_{1} \\
\vdots \\
u_{N}
\end{array}\right]
$$

The FETI method assumes that Dirichlet boundary conditions are inherited from the original problem, which is shown in fig. 2(a). This fact implies that the magnitudes of defects of the stiffness matrices, $K_{i}$, may vary from zero, for the subdomains with enough Dirichlet conditions, to the possible maximum (6 for 3D solid mechanics problems and 3 for $2 \mathrm{D}$ ones) in the case of the sub-domains exhibiting some rigid body modes. General solution to such systems requires computation of generalised inverses and bases of the null spaces, i.e. kernels, of the underlying singular matrices. The problem is that the magnitudes of the defects are difficult to evaluate because this computation is extremely disposed to the round off errors; see Farhat and Géradin [8].

\section{Total FETI method}

In this section we briefly review the main ideas the TFETI method stems from. To circumvent the problem of computing bases of the kernels of singular matrices, Dostál [5] came up with a novel solution. His idea was to remove all the prescribed Dirichlet boundary conditions and to enforce them by additional Lagrange multipliers denoted as $\lambda^{B}$ in fig. 2(b). The effect of the procedure on the stiffness matrices of the sub-domains is that their defects are the same and their magnitude is known beforehand. From the computational point of view such approach is advantageous; see [8] for discussion of this topic.

The overall approach resembles the classic one by Farhat et al. [2] and others, e.g. [3]. The Lagrangian associated with the problem governed by eqn (1) is as reads

$$
L(u, \lambda)=\frac{1}{2} u^{\top} K u-f^{\top} u+\lambda^{\top} B u .
$$

This is equivalent to the saddle point problem

$$
\text { Find }(\bar{u}, \bar{\lambda}) \quad \text { so that } \quad L(\bar{u}, \bar{\lambda})=\sup _{\lambda} \inf _{u} L(u, \lambda) \text {. }
$$


For $\lambda$ fixed, the Lagrangian $L(., \lambda)$ is convex in the first variable and a minimiser $u$ of $L(., \lambda)$ satisfies the following equation

$$
K u-f+B^{\top} \lambda=0 .
$$

Eqn (5) has a solution if and only if $f-B^{\top} \lambda$ belongs to the range of $K$ and therefore the following relationship holds

$$
R^{\top}\left(f-B^{\top} \lambda\right)=0 .
$$

$R$ denotes the full rank matrix with columns spanning the kernel of $K$. The kernels of the sub-domains are known and can be assembled directly.

It is necessary to eliminate the primal variable $u$ from eqn (5). It may be easily verified that if $u$ is a solution to eqn (5), then there exists a vector $\alpha$ such that

$$
u=K^{\dagger}\left(f-B^{\top} \lambda\right)+R \alpha
$$

where $K^{\dagger}$ is any symmetric positive definite matrix satisfying $K K^{\dagger} K=K$.

Substituting eqn (7) into eqn (4), we get the following minimisation problem

$$
\min \frac{1}{2} \lambda^{\top} B K^{\dagger} B^{\top} \lambda-\lambda^{\top} B K^{\dagger} f, \quad \text { s.t. } \quad R^{\top}\left(f-B^{\top} \lambda\right)=0 .
$$

Let us introduce notations

$$
F=B K^{\dagger} B^{\top}, \quad G=R^{\top} B^{\top}, \quad e=R^{\top} f, \quad d=B K^{\dagger} f,
$$

so that the problem (8) reads

$$
\min \frac{1}{2} \lambda^{\top} F \lambda-\lambda^{\top} d \quad \text { s.t. } \quad G \lambda=0 .
$$

The final step stems from observation that the problem (10) is equivalent to

$$
\min \frac{1}{2} \lambda^{\top} P F P \lambda-\lambda^{\top} P d \quad \text { s.t. } \quad G \lambda=0,
$$

where

$$
P=I-Q \quad \text { and } \quad Q=G^{\top}\left(G G^{\top}\right)^{-1} G
$$

stand for the orthogonal projectors on the kernel of $G$ and the image space of $G^{\top}$, respectively.

The problem (11) may be solved efficiently by the conjugate gradient method because the estimate of the spectral condition number for the FETI method also holds for the TFETI method [5].

It was shown that application of the TFETI methodology to the contact problems converts the original problem to the quadratic programming one with simple bounds and equality constraints. This problem can be further transformed by Semi-Monotonic Augmented Lagrangians with Bound and Equality constraints (SMALBE) method to the sequence of simply bounded quadratic programming 
problems. These auxiliary problems may be solved efficiently by the Modified Proportioning with Reduced Gradient Projection (MPRGP) method. The detailed descriptions of SMALBE and MPRGP are beyond the scope of this paper and can be found in Dostál [9]. It was proved in Dostál [10] that application of combination of both these methods to solution to contact problems benefits the numerical and parallel scalabilities.

\section{Non-linear algorithm}

Herein we extend application of the FETI and TFETI methods to problems with the geometric and material non-linearities. The algorithm based solely on the assumptions and relationships presented in the previous Sections is directly applicable to solution to the contact problems, but with other conditions linear. Any additional non-linear effect necessitates employment of the nested iteration strategy, where the inner loop accounts for TFETI based contact solver while the outer loop is concerned with the material and geometric non-linear effects, contact geometry update and equilibrium iterations.

The state of equilibrium is characterised by condition that the internal forces equal the total external forces, i.e., the residual equals zero

$$
r e s=f_{\text {ext }}-f_{\text {int }}=0 .
$$

The total external forces consist of the applied external forces and the contact ones

$$
f_{e x t}=f-B^{\top} \lambda .
$$

The internal forces can be expressed as follows

$$
f_{\text {int }}=\sum_{\text {nelem }} \int_{V_{e}} B_{s}^{\top}(\epsilon) \sigma(\epsilon) d V
$$

where $B_{s}$ is an appropriate element matrix relating the element strain tensor $\epsilon$ with nodal displacements while considering the geometric non-linearities, and $\sigma$ is the element stress tensor arising in general from non-linear material behaviour. We sum over the total number of elements nelem.

The solution algorithm is shown in the following flowchart.

Initial step: Assemble stiffness matrix $K=\operatorname{diag}\left\{K_{1}, \ldots, K_{p}\right\}$ and $B^{E}$; Set $\quad i=0 ; \quad \mathrm{u}^{0}=0, \quad \lambda^{0}=0, \quad f_{\text {int }}^{0}=0$;

Step 1: Evaluate contact conditions $B^{I i}$;

Step 2: Solve contact problem for $\Delta \lambda \rightarrow \Delta \mathrm{u}$;

Step 3: $\lambda^{i}=\lambda^{i-1}+\Delta \lambda, \quad \mathrm{u}^{i}=\mathrm{u}^{i-1}+\Delta \mathrm{u}$; $f_{\text {int }}^{i}=\sum_{\text {nelem }} \int_{V_{e}} B_{s}^{\top}\left(\epsilon^{i}\right) \sigma\left(\epsilon^{i}\right) d V$

Assemble residual load vector $r e s^{i}=f-B^{i \top} \lambda^{i}-f_{i n t}^{i}$;

Check on convergence criteria $\quad \frac{\|\Delta \mathrm{u}\|}{\left\|\mathrm{u}^{i}\right\|}<\eta_{1}, \quad \frac{\left\|r e s^{i}\right\|}{\left\|f_{e x t}^{i}\right\|}<\eta_{2} ;$ 
If fulfilled then STOP, otherwise set $i \leftarrow i+1$ and go to Step 1

Step 2 represents the inner iteration loop for evaluation of the Lagrange multipliers enforcing compatibility between the sub-domains along both fictitious and contact interfaces and at nodes with the applied Dirichlet conditions.

\section{Numerical experiments}

To demonstrate the ability of our algorithms to solve contact problems, we show results of two numerical experiments. The first case is concerned with contact problem of two cylinders, and the second one with contact problem of the pin in hole with small clearance.

\subsection{Contact problem of two cylinders}

Consider contact of two cylinders with parallel axes. We can consider only one half of the problem due to its symmetry. The diameter of the upper cylinder $R_{u}=1 \mathrm{~m}$ and of the lower one $R_{l}=\infty$. In spite of the fact that it is the 2D problem, it is modelled with $3 \mathrm{D}$ continuum tri-linear elements with two layers of them along the axis of symmetry of the upper cylinder. The model consists of 8904 elements and 12765 nodes. The boundary conditions are imposed in such a way that they generate, from the physical point of view, the plane strain problem. The material properties are as follows: Young's modulus $E=2.0 \times 10^{11} \mathrm{~Pa}$ and Poisson's ratio $\nu=0.3$.

First, the upper cylinder is loaded by $40 \mathrm{MN} / \mathrm{m}$ along its upper line and the problem is considered linearly elastic and linearly geometric. Fig. 3(a) shows solution in terms of the deformed mesh.

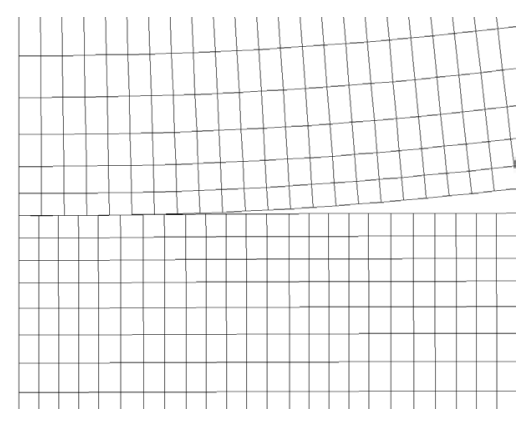

(a) Linear problem.

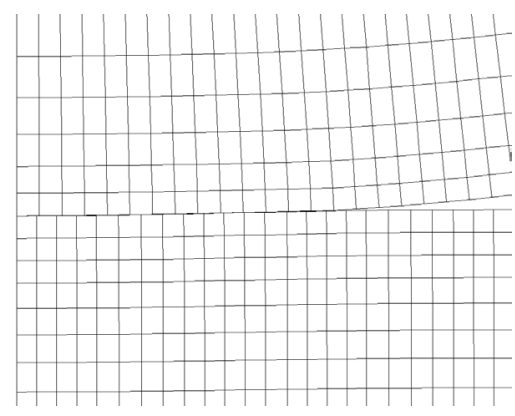

(b) Non-linear problem.

Figure 3: The problem of two cylinders, deformed meshes. 
Next, the problem was computed on the same mesh with the same loading, but we considered the linearly-elastic-perfectly-plastic material model with the yield stress $\sigma_{Y}=800 \mathrm{MPa}$. We also considered the geometric non-linearity. The deformed mesh is depicted in fig. 3(b).

In the latter case we iterated in the outer loop in the sense of the solution algorithm described in Section 4. The number of the outer iterations was 15. The number of iterations of the MPRGP algorithm for contact evaluation at each cycle of the outer loop is shown in fig. 4.

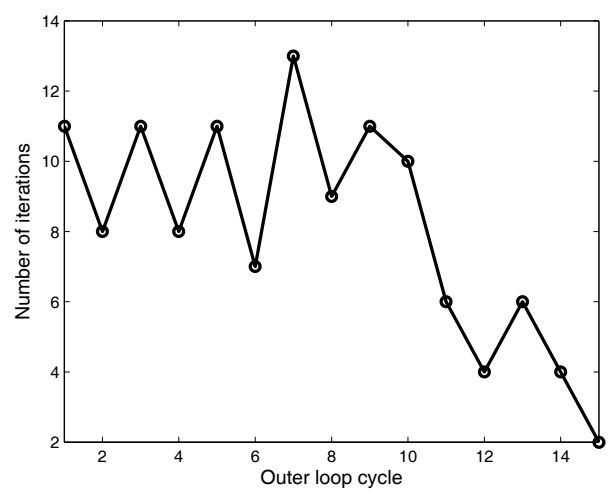

Figure 4: Number of MPRGP iterations at each cycle of the outer loop.

\subsection{Pin-in-hole contact problem}

Consider a problem of the circular pin in circular hole with small clearance. The radius of the hole is $1 \mathrm{~m}$ and the pin has its radius $1 \%$ smaller. Again, the 2D problem is modelled with 3D elements. The model consists of 15844 tri-linear elements and 28828 nodes. The pin is loaded along its centre line by $133 \mathrm{MN} / \mathrm{m}$. The geometric non-linearity was considered. The material properties are the same as in the previous case.

Fig. 5 shows the normal contact stress distribution along surface of the pin from the plane of symmetry. The distribution of this stress along the hole is practically identical.

\section{Conclusion}

Application of a new variant of the FETI domain decomposition method to solution to contact problems with additional non-linear effects was presented. It is called the Total FETI method, and its basic idea, in comparison with the FETI method, consists in replacement of the Dirichlet boundary conditions by the Lagrange multipliers with physical meaning of forces in this context. This feature 


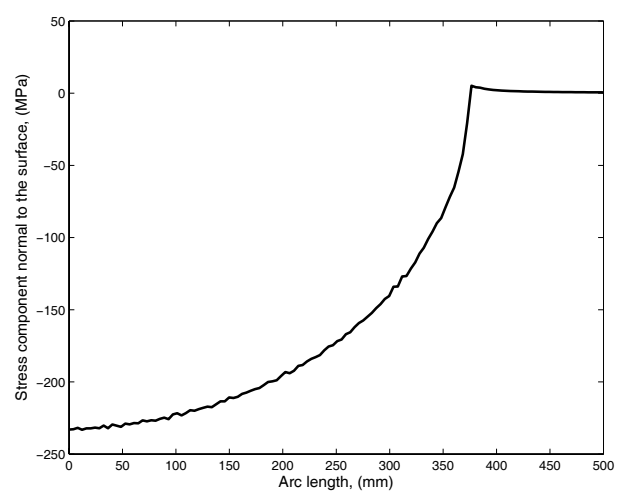

Figure 5: The pin-in-hole problem, normal contact stress on the pin, geometrically non-linear case.

is of great importance from the computational point of view, because the magnitudes of defects of stiffness matrices of all the sub-domains are the same and their magnitude is known beforehand. The numerical experiments show that algorithm stemming from the TFETI method is applicable to solution to contact problems accompanied by other non-linearities.

\section{Acknowledgement}

The authors would like to acknowledge the support of GA CR through grant number 101/05/0423 and AS CR through grant number AV0Z20760514.

\section{References}

[1] Farhat Ch. \& Roux F.X., A method of finite element tearing and interconnecting and its parallel solution algorithm. International Journal for Numerical Methods in Engineering, 32(12), pp. 1205-1227, 1991.

[2] Farhat Ch., Mandel J. \& Roux F.X., Optimal convergence properties of the FETI domain decomposition method. Computer Methods in Applied Mechanics and Engineering, 115(5), pp. 365-385, 1994.

[3] Dostál Z., Horák D., Kučera R., Vondrák V., Haslinger J., Dobiáš J. \& Pták S., FETI based algorithms for contact problems: scalability, large displacements and 3D Coulomb friction. Computer Methods in Applied Mechanics and Engineering, 194(2-5), pp. 395-409, 2005.

[4] Dostál Z. \& Horák D., Scalable FETI with optimal dual penalty for a variational inequality. Numerical Linear Algebra with Applications, 11(6), pp. 455-472, 2004. 
[5] Dostál Z., Horák D. \& Kučera R., Total FETI - an easier implementable variant of the FETI method for numerical solution of elliptic PDE. To be published in Communications in Numerical Methods in Engineering.

[6] PMD manuals, www.it.cas.cz/manual/pmd

[7] Laursen T.A., Computational contact and impact mechanics, SpringerVerlag: Berlin, pp. 8-17, 2002.

[8] Farhat Ch. \& Géradin M., On the general solution by a direct method of a large-scale singular system of linear equations: application to the analysis of floating structures. International Journal for Numerical Methods in Engineering, 41(7), pp. 675-696, 1998.

[9] Dostál Z. \& Schöberl J., Minimizing quadratic functions over non-negative cone with the rate of convergence and finite termination. Computational Optimization and Application, 30(1), pp. 23-43, 2005.

[10] Dostál Z., Inexact semi-monotonic augmented Lagrangians with optimal feasibility convergence for convex bound and equality constrained quadratic programming. SIAM Journal on Numerical Analysis, 43(2), pp. 96-115, 2005. 